\title{
Le réseau de mesures SYVEL : un outil performant et indispensable à la compréhension et au suivi du fonctionnement de l'estuaire de la Loire
}

\author{
Hélène FALLOU
}

GIP Loire Estuaire, 22 rue de la Tour d'Auvergne 44200 Nantes

02.51.72.93.66

gip@loire-estuaire.org

\section{INTRODUCTION}

Le territoire d'études du GIP Loire Estuaire se situe au débouché du bassin versant de la Loire, entre la Maine et l'océan Atlantique, sur une distance d'environ $150 \mathrm{~km}$, des Ponts-de-Cé à Saint-Nazaire. Une partie de ce territoire est soumise à l'influence de la marée. L'estuaire de la Loire est un milieu complexe où se mélangent les eaux douces du fleuve et salées de l'océan. Un estuaire dynamique est ainsi défini jusqu'à Ancenis, à près de $100 \mathrm{~km}$ de Saint-Nazaire.

Il comprend une zone de forte turbidité (aussi appelée « bouchon vaseux » qui se caractérise par une forte concentration en matières en suspension), des gradients de salinité, de température et d'oxygène dissous. La variabilité de ces phénomènes est forte et dépend en grande partie des apports en eau douce (débit du fleuve) et de la dynamique marine (coefficient de marée).

Le réseau de mesures SYVEL (SYstème de Veille dans l'Estuaire de la Loire), propriété du GIP Loire Estuaire a été mis en place en 2006, pour un début opérationnel en janvier 2007. Les objectifs de ce réseau sont multiples. Il vise à suivre l'évolution à long terme des grands paramètres estuariens (salinité, oxygène dissous, matières en suspension), à quantifier les impacts des aménagements potentiels et à comprendre les différents processus mis en jeu.

Il s'agit de mesurer les paramètres en lien avec les usages et la qualité de l'eau. Le suivi du front de salinité, défini pour une concentration en sel de $0,5 \mathrm{~g} / \mathrm{l}$, est essentiel pour la gestion de l'alimentation en eau potable de l'agglomération de Nantes. La localisation du bouchon vaseux et des zones d'hypoxie (concentration en oxygène dissous inférieure à 5 $\mathrm{mg} / \mathrm{l}$ ) sont des éléments importants pour suivre la qualité des eaux et son influence sur la vie aquatique.

Il est également utile de pouvoir quantifier les flux sédimentaires qui proviennent de l'amont, mais aussi ceux qui arrivent à la mer.

Le réseau est donc utile pour la connaissance des mécanismes estuariens, et les liens avec les activités professionnelles d'un certain nombre d'acteurs sur l'estuaire (exploitation agricole, industrielle, gestionnaires des milieux naturels...).

Ce réseau est composé de six stations (dont une est la propriété d'EDF), localisées dans la partie aval de l'estuaire, entre l'amont de Nantes et Donges (sur $53 \mathrm{~km}$ ). Les stations amont de Bellevue et Trentemoult assurent un suivi précis de la limite du front de salinité et des zones turbides. Les stations du Pellerin, de Cordemais et de Paimboeuf permettent d'apporter des précisions sur la localisation du bouchon vaseux. Ces stations sont également nécessaires pour le suivi des zones d'hypoxie, leur durée et leur intensité. La station de Donges, la plus aval du réseau, a été choisie pour observer la distribution de la salinité et des flux sédimentaires. Cette station est également équipée d'un deuxième point de mesure, à une profondeur de -4 mètres, afin d'apprécier les phénomènes de stratification verticale.

Quatre paramètres sont suivis en continu, à une fréquence de 10 à 15 minutes ( 1 heure pour Cordemais) : la température, la turbidité (concentration en matières en suspension), la conductivité (salinité), la concentration en oxygène dissous. Les stations mesurent les différents paramètres en sub-surface (1 mètre de profondeur).

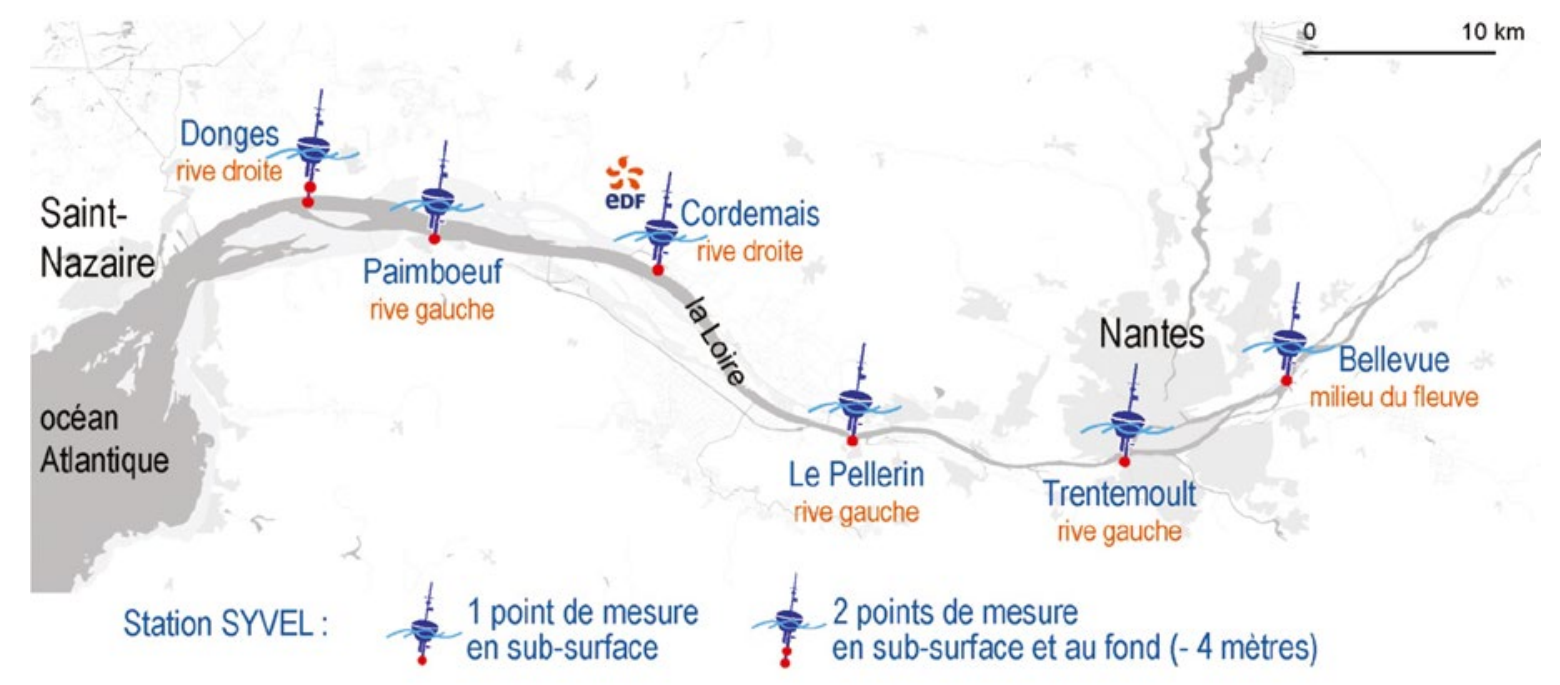

Figure 1 : Carte des stations du réseau de mesures SYVEL 
Deux technologies sont actuellement en place sur le réseau SYVEL. Pour les stations de Trentemoult, Le Pellerin, Paimboeuf et Donges, des stations estuariennes de type MAREL sont installées. Elles permettent de prélever l'eau dans la Loire, et de l'acheminer vers une chambre de mesures où sont situés les différents capteurs. La station de Bellevue, installée sur une pile de pont, est équipée d'une sonde multi-paramètres SMATCH. Ces capteurs, autonomes en énergie, sont directement immergés dans la Loire. Les données de l'ensemble des stations sont envoyées par GPRS, sur un poste de gestion dédié dans les bureaux du GIP Loire Estuaire.

\section{UN NOMBRE DE STATIONS COHÉRENT POUR SUIVRE LE FONCTIONNEMENT DE L'ESTUAIRE DE LA LOIRE}

Dans l'estuaire, il existe des gradients pour les différents paramètres. La salinité présentée sur la Figure 2 est mesurée à l'heure de la pleine mer à Donges. Des variations importantes de 0,5 à $26 \mathrm{~g} / 1$ sont observées entre Nantes et Donges. Ainsi, une seule station ne permet pas de représenter l'ensemble de la masse d'eau, pour un instant donné. Afin d'avoir une vision plus complète du fonctionnement de l'estuaire, il est nécessaire d'avoir un réseau avec ces 6 stations.

Le bouchon vaseux est un phénomène naturel des estuaires macrotidaux (à fort marnage) comme l'estuaire de la Loire. Il se compose de sédiments fins en suspension mêlés de matière organique qui s'accumulent dans la zone de mélange des eaux douces et salées. Il peut être visible entre Mauves-sur-Loire et l'aval de Saint-Nazaire au maximum de son étendue. La localisation du bouchon vaseux est soumise à l'influence du débit du fleuve. Plus le débit du fleuve est faible, plus le bouchon vaseux a tendance à remonter à l'amont de l'estuaire, et plus son emprise est grande. A l'inverse, en période de crues, le bouchon vaseux sera visible à l'aval et une partie pourra être expulsée de l'estuaire. De plus, pour un même débit, plus les coefficients de marée sont importants, plus la remise en suspension des particules est importante et plus le bouchon vaseux sera étendu.
Par exemple, pour un débit d'étiage de $200 \mathrm{~m}^{3} / \mathrm{s}$, et des vives-eaux (coefficient supérieur à 90), le bouchon vaseux est visible entre l'amont de Bellevue et l'aval de Donges, avec un panache qui s'étend de Mauves-sur-Loire à Saint-Nazaire. Ce panache est mesuré par d'autres réseaux de mesure présents sur la Loire, avec une fréquence d'une mesure par mois.

Aussi, afin de définir avec précision la localisation de ce bouchon vaseux, il est essentiel d'avoir des stations de mesure réparties régulièrement dans l'estuaire.

\section{UN RÉSEAU DE MESURES HAUTE FRÉQUENCE POUR SUIVRE DES PHÉNOMÈNES PRÉSENTANT DE GRANDES VARIATIONS TEMPORELLES}

Afin de suivre des phénomènes présentant une grande variabilité temporelle, il est essentiel d'avoir un réseau de mesures haute fréquence. En effet, l'estuaire est soumis à des cycles de marée à différentes échelles.

Des cycles lunaires de marée entrainent des variations des coefficients de marée. Lors des forts coefficients (vives-eaux), les concentrations en oxygène dissous ont tendance à diminuer, sous l'effet de la remise en suspension des sédiments. Au contraire, lorsque les coefficients de marée diminuent (mortes-eaux), les concentrations en oxygène dissous augmentent. Ainsi, une mesure par mois apporte une partie de l'information, mais ne permet pas d'observer ces variations mises en évidence par les suivis haute fréquence.

A ces cycles de vives-eaux / mortes-eaux, s'ajoutent les marées journalières qui influencent les différents paramètres. Les variations des concentrations en oxygène sont principalement liées au transport de la masse d'eau. L'oxygène est consommé dans le bouchon vaseux, situé le plus souvent à l'aval. Le flot transporte alors la masse d'eau désoxygénée vers l'amont, provoquant une diminution de la concentration en oxygène, à pleine mer locale. Au contraire, à l'aval les concentrations les plus faibles sont observées à basse mer locale. Une mesure par jour, ou même une mesure par heure, ne permet pas d'observer les pics d'oxygénation ou d'hypoxie.

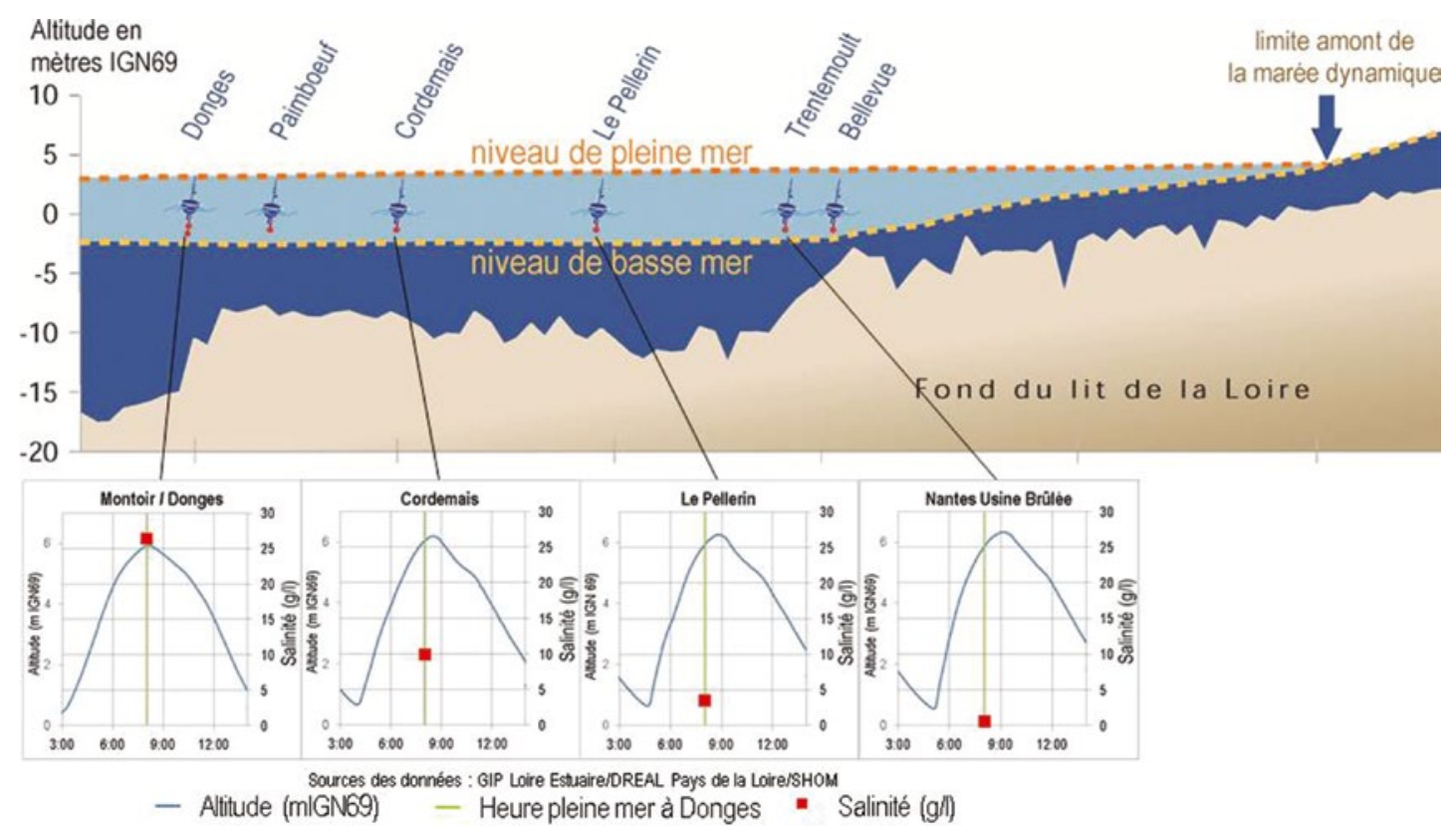

Figure 2 : Mesures de la salinité le 04 août 2015, à $08 h 02$ (débit à Montjean-sur-Loire : $141 \mathrm{~m}^{3} / \mathrm{s}$; coefficient de marée : 
Un réseau de mesures haute-fréquence avec une mesure toutes les 10 minutes permet d'observer des évènements fugaces et non prévisibles. A titre d'exemple, lors de la tempête Xynthia, le 28 février 2010, la combinaison de vents forts et de grandes marées (coefficient supérieur à 100), a entraîné la remontée de l'océan dans le fleuve. Au niveau de la station de Cordemais, un pic de salinité a été observé pendant cinq heures. La concentration maximale mesurée atteint $6 \mathrm{~g} / \mathrm{l}$, alors que la salinité attendue était de $1 \mathrm{~g} / \mathrm{l}$.

\section{RETOURS SUR 10 ANS DE FONCTIONNEMENT}

Depuis l'installation du réseau, en 2007 , le taux de fonctionnement sur l'ensemble des stations atteint plus de $80 \%$.

Chaque jour, 5184 données arrivent, par télétransmission, sur un poste de gestion dédié. Elles sont validées et intégrées à une base de données développée spécifiquement pour le réseau SYVEL, puis analysées et/ou diffusées pour répondre aux problématiques posées par les acteurs de la Loire.

Deux niveaux de validation ont été élaborés. Le traitement de niveau 1 a pour objectif de qualifier visuellement les données de " bonnes », " douteuses » ou " fausses ». Les données produites à ce stade sont dédiées à la surveillance du milieu (alerte). Le traitement de niveau 2 a pour objectif de produire une donnée à vocation scientifique (expertise). Il est réalisé après la calibration des capteurs, afin de corriger les éventuelles dérives.

Sur les dix ans de fonctionnement du réseau, l'évolution des paramètres estuariens est dominée par les apports en eau douce du fleuve, imprévisibles et changeants qui rencontrent les apports océaniques souvent prévisibles et cycliques; les deux sont largement influencés par les conditions météorologiques qui ajoutent de la complexité dans la compréhension et le suivi de l'évolution de la température, du bouchon vaseux, de la salinité et de l'oxygénation des eaux estuariennes.

Entre 2007 et 2016, la température de l'eau a été marquée par des hivers froids en février 2012, avec des températures proches de $0^{\circ} \mathrm{C}$, et des étés chauds en 2009 et 2013 .

La salinité est elle aussi soumise aux conditions hydrologiques. Les étiages prolongés entrainent des salinités plus importantes au niveau des stations de Cordemais et du Pellerin. Le front de salinité (à $0,5 \mathrm{~g} / \mathrm{l})$ n'a pas été mesuré à la station de Bellevue pendant les 10 années de mesure du réseau SYVEL.

De la même manière, l'évolution de la position et des concentrations du bouchon vaseux est dominée principalement par le débit du fleuve. Lors des années très sèches (2011 et 2015), des concentrations en matières en suspension supérieures à $0,5 \mathrm{~g} / 1$ sont observées plus d'un tiers du temps, à la station du Pellerin.

Concernant l'évolution des zones et des périodes d'hypoxie, la variation des conditions hydrologiques est le principal facteur explicatif. En 2011, l'étiage précoce et prolongé et les températures élevées dès le printemps ont entrainé des déficits en oxygène dissous dès le mois d'avril. Des hypoxies ont également été observées dès le mois de mai sur la station de Trentemoult. Au contraire, des années humides comme 2013, présentent des hypoxies sur des périodes plus courtes. La température joue également un rôle important sur l'intensité des hypoxies. Les années où les températures de l'eau sont plus élevées, les concentrations en oxygène dissous sont plus faibles. La durée, l'étendue et l'intensité des crises d'hypoxie ont un impact sur le comportement des poissons. Les effets varient en fonction des espèces et de leur stade de développement. Les conséquences peuvent aller d'effets négatifs sur la croissance ou de retard migratoire, jusqu'à la létalité.

\section{CONCLUSIONS}

Pour observer une tendance dans l'estuaire de la Loire, encore plus que dans tout autre écosystème, il faut disposer de longues séries chronologiques de données, sur un nombre suffisant de stations de mesure tant la variabilité des paramètres est grande sur les $100 \mathrm{~km}$ d'estuaire. De plus, la compréhension reste partielle car le réseau SYVEL n'est actuellement équipé que de stations qui mesurent en subsurface. Or les paramètres varient également sur la verticale (stratification de l'estuaire). Seule la station de Donges est équipée d'un second point de mesure en profondeur $(-4 \mathrm{~m})$, afin d'observer et de suivre une partie de la stratification verticale. A titre d'exemple, les eaux profondes sont plus salées qu'en surface, et d'autant plus que les années sont sèches ; également, les sédiments se déposant sur le fond du fleuve, les concentrations en matières en suspension sont plus importantes au fond qu'en surface, de même, les déficits en oxygène dissous sont plus marqués au fond qu'en surface.

L'exploitation et la valorisation des données des réseaux sont essentielles pour mieux appréhender le fonctionnement des estuaires et leur évolution. Les données alimentent les modélisations mathématiques, les expertises piscicoles, les programmes de recherche et bénéficient, entre autres, aux gestionnaires de l'eau, des espaces naturels et de la voie navigable.

Les échanges entre les différents estuaires permettent d'améliorer la compréhension des différents phénomènes. Les estuaires de la Seine et de la Gironde sont également équipés de réseaux haute-fréquence qui mesurent notamment la température de l'eau, la turbidité, la concentration en oxygène dissous et la salinité. Leurs objectifs sont similaires à ceux du réseau SYVEL.

Des différences de comportement sont observées entre les trois estuaires. Alors que les estuaires de la Loire et de la Gironde sont régulièrement soumis à des crises d'hypoxie, dans l'estuaire de la Seine aucun déficit en oxygène (concentration inférieure à $3 \mathrm{mg} / \mathrm{l}$ ) n'a été mesuré en subsurface depuis 2007. 